\title{
Brain reactivity and selective attention to sleep-related words in patients with chronic insomnia
}

\author{
Kai Spiegelhalder ${ }^{\mathrm{a}, *}$, Chiara Baglioni ${ }^{\mathrm{a},{ }^{*}}$, Wolfram Regen ${ }^{\mathrm{a}}$, Simon D. Kyle ${ }^{\mathrm{b}}$, \\ Christoph Nissen $^{\mathrm{a}}$, Jürgen Hennig ${ }^{\mathrm{c}}$, John-Peter Doerr ${ }^{\mathrm{a}}$, Bernd Feige ${ }^{\mathrm{a}}$, and Dieter Riemann ${ }^{\mathrm{a}}$ \\ a Clinic for Psychiatry and Psychotherapy, Medical Center - University of Freiburg, Faculty \\ of Medicine, University of Freiburg, Germany, Germany \\ b Sleep and Circadian Neuroscience Institute (SCNi), Nuffield Department of Clinical \\ Neuroscience, University of Oxford, UK \\ c Department of Diagnostic Radiology, Medical Center - University of Freiburg, Faculty of \\ Medicine, University of Freiburg, Germany, Germany \\ * These authors contributed equally to this work.
}

Total number of figures: 1 (+2 supplemental figures)

Total number of tables: 3

Financial support: This was not an industry supported study.

Conflicts of interest disclosure: K. Spiegelhalder, C. Baglioni, W. Regen, J. Hennig, J.P. Doerr, B. Feige, and D. Riemann declare they have no conflict of interest. S. D. Kyle has been involved in research with Big Health/Sleepio Ltd. and has acted as consultant for Big Helath/Sleepio Ltd., and C. Nissen has received speaker honoraria from Servier and Vanda Pharmaceuticals.

\section{Correspondence to:}

Kai Spiegelhalder, MD PhD

University of Freiburg Medical Center

Department of Psychophysiology/ Sleep Medicine

Clinic of Psychiatry and Psychotherapy

Hauptstraße 5, 79104 Freiburg, GERMANY

Tel.: +49 $761270-65890$

Fax: +49 761 270-66190

E-Mail: Kai.Spiegelhalder@uniklinik-freiburg.de 


\begin{abstract}
Objective/Background: Sleep-related attentional bias has been suggested to represent an important factor for the maintenance of chronic insomnia. However, little is known about potentially underlying psychological mechanisms such as threat or craving. As these are associated with distinguishable brain activation patterns, we performed a functional neuroimaging study.
\end{abstract}

Participants/Methods: Functional magnetic resonance imaging was used to investigate brain reactivity to sleep-related words in 20 patients with primary insomnia according to DSM-IV criteria and 35 good sleeper controls according to Research Diagnostic Criteria. In addition, an emotional Stroop task was performed in all participants outside the scanner to investigate sleep-related attentional bias.

Results: Contrary to the hypotheses, patients with chronic insomnia did not differ from good sleeper controls in terms of brain reactivity and selective attention to sleep-related words. In addition, exploratory analyses did not show any significant correlations between brain reactivity/ selective attention to sleep-related words and questionnaire scores/ PSG parameters.

Conclusions: The results from the present study call into question that attentional bias to sleep-related stimuli is a core feature of chronic insomnia. Future studies may use pictorial stimuli and larger sample sizes for investigating sleep-related information processing in insomnia.

Keywords: Attentional bias; brain imaging; cognitive bias; emotional Stroop task; functional magnetic resonance imaging; insomnia. 


\section{Introduction}

Sleep-related attentional bias is defined as the tendency to selectively attend to sleep-related information in comparison to neutral information and has been suggested to represent an important factor for the maintenance of chronic insomnia (Espie, Broomfield, MacMahon, Macphee, \& Taylor, 2006). The primary evidence for this claim comes from studies using computerised reaction time tasks in which selective attention to sleep-related stimuli affects performance in individuals with clinical or subclinical insomnia (Barclay \& Ellis, 2013; Jansson-Fröjmark, Bermas, \& Kjellen, 2013; Jones, Macphee, Broomfield, Jones, \& Espie, 2005; Lundh, Fröding, Gyllenhammar, Broman, \& Hetta, 1997; MacMahon, Broomfield, \& Espie, 2006; Spiegelhalder, Espie, Nissen, \& Riemann, 2008; Spiegelhalder, Espie, \& Riemann, 2009; Spiegelhalder et al., 2010; Taylor, Espie, \& White, 2003; Woods, Marchetti, Biello, \& Espie, 2009). These tasks involve the presentation of sleep-related and control stimuli, such as sleep-related and neutral pictures or words. We have previously validated a set of sleep-related and control words that showed a sleep-related attentional bias in patients

with insomnia compared to good sleeper controls (Spiegelhalder et al., 2010), and a significant association between poor sleep quality and sleep-related attentional bias in a nonclinical sample (Spiegelhalder et al., 2009). The most widely used task that is based on the presentation of words is the emotional Stroop task which measures the degree to which participants are slower in responding to the colour of concern-related words than to the colour of neutral words (Williams, Mathews, \& MacLeod, 1996).

What is yet to be determined is what 'drives' sleep-related attentional bias. Espie et al. (2006) suggest that, unlike anxiety, the poor sleeper may, on viewing sleep-related stimuli, demonstrate signs of craving, perhaps in the same way one might towards food following a period of food deprivation. Thus, sleep cues may have some incentive value in a similar manner as substances do for individuals with substance dependence. This is in line with 
previous studies using facial electromyography which suggested a positive emotional response to sleep-related pictures in those with insomnia (Baglioni et al., 2010; Lombardo et al., 2013). The inverse is also possible: sleep-cues may represent a threat to the poor sleeper, invoking anxiety (Harvey, 2002). Patients with chronic insomnia do report impairments in health-related quality of life and several domains of daytime functioning, as well as marked distress during the pre-sleep period. As a consequence of this, it is likely that sleep-related stimuli may also have an aversive quality.

Up to now, only few empirical studies have contributed to our understanding of psychological mechanisms underlying selective sleep-related attention, and the results are inconclusive. Both the positive association between sleep-related attentional bias and markers of improved sleep continuity in the subsequent night (Spiegelhalder et al., 2010) and the positive association between sleep-related attentional bias and state sleepiness in healthy individuals (Spiegelhalder et al., 2009) support the 'craving hypothesis'. However, the study by Spiegelhalder et al. (2009) also reported a positive association between sleep-related attentional bias and a trait marker of poor sleep quality which was independent of state sleepiness. Moreover, Sagaspe et al. (2006) did not find any significant relationship between state sleepiness and sleep-related attentional bias. These findings may be regarded as supporting the 'threat hypothesis' of sleep-related attentional bias.

As threat and craving are associated with distinguishable brain activation patterns (Etkin \& Wagner, 2007; Schacht, Anton, \& Myrick, 2012), functional magnetic resonance imaging (fMRI) may help us to elucidate the mechanisms underlying sleep-related attentional bias. In a recently published fMRI study (called "emo-fMRI experiment"), we reported increased amygdala reactivity to insomnia-specific stimuli in patients with chronic insomnia suggesting anxiety-related brain activity in response to sleep-related information (Baglioni et al., 2014a). However, the stimuli of this study were comprised of pictures of individuals lying awake in 
bed at night, and, thus, were specifically related to the experience of insomnia and had an inherently negative valence. Moreover, the region of interest-based analysis was restricted to the amygdala. Therefore, this study was not designed to discriminate between the potential roles of threat and craving for sleep-related attentional processes.

The current study aimed at elucidating the psychological mechanisms of sleep-related attentional bias by using fMRI to investigate brain reactivity to sleep-related stimuli in patients with chronic insomnia and good sleeper controls. The stimulus set was comprised of sleep-related and control words that have been previously validated (Spiegelhalder et al., 2008; 2009; 2010). After the current fMRI experiment (called "sleep-fMRI experiment"), participants performed an emotional Stroop task outside the scanner using the same set of sleep-related and control words. We hypothesised that patients with chronic insomnia would show altered brain reactivity and selective attention to sleep-related words. The functional neuroimaging data was first analysed using a whole-brain corrected level of significance. Subsequently, exploratory region-of-interest analyses were carried out for anxiety-related brain areas, namely the left and right amygdala and insula (Etkin \& Wagner, 2007), as well as for craving-related brain areas, namely the left and right ventral striatum and posterior cingulate cortex (PCC; Schacht et al., 2012).

\section{Material and methods}

\section{Participants}

Participants consisted of 25 patients with primary insomnia according to DSM-IV criteria (American Psychiatric Association, 2000) and 40 good sleeper controls according to the Research Diagnostic Criteria for good sleepers (Edinger et al., 2004; other findings from this 
sample have been published elsewhere: Baglioni et al., 2014a; Spiegelhalder et al., 2013; 2014). All patients with primary insomnia who were referred to our sleep disorders clinic by their primary care providers between October 2008 and June 2011 were screened systematically for study participation. Good sleeper controls were recruited through local advertisements. Before entering the protocol, all participants underwent our standard physical and psychiatric examination including a semi-standardised psychiatric and sleep-related interview conducted by an experienced psychiatrist, excluding those with medical, psychiatric or occult sleep disorder pathology. All participants were right-handed and free of any psychoactive medication at least 2 weeks prior to and during the study. Two control participants were excluded from the analysis because of pathological MRI scans (internal hydrocephalus, brain damage after traumatic brain injury); two control participants and one patient with chronicinsomnia were excluded due to missing data resulting from technical problems during the fMRI acquisition; one control participant and four patients with chronic insomnia were excluded because they had more than $10 \%$ omissions in the simple reaction time task that was used to ensure participants' attention during the sleep-fMRI experiment (see below). Thus, the final sample consisted of 20 patients with chronic insomnia and 35 good sleeper controls. All participants gave their informed written consent prior to inclusion

in the study. The study protocol was approved by the Institutional Review Board of the University Medical Center Freiburg and conducted in accordance with the Declaration of Helsinki.

\section{Questionnaires}

The detailed psychometric assessment of the sample included the Insomnia Severity Index (ISI; Bastien, Vallières, \& Morin, 2001), the Pittsburgh Sleep Quality Index (PSQI; Buysse, Reynolds, Monk, Berman, \& Kupfer, 1989), the brief version of the Dysfunctional Beliefs 
and Attitudes about Sleep Scale (DBAS-16; Morin, Vallières, \& Ivers, 2007), the Glasgow Sleep Effort Scale (GSES; Broomfield \& Espie, 2005), the Pre-Sleep Arousal Scale (PSAS; Nicassio, Mendlowitz, Fussell, \& Petras, 1985), the Epworth Sleepiness Scale (ESS; Johns, 1991), the Beck Depression Inventory (BDI; Beck \& Steer, 1987), and the trait subscale of the State-Trait Anxiety Inventory (STAI; Spielberger, Gorsuch, Lushene, Vogg, \& Jacobs, 1983). In addition, participants filled in the state version of the STAI (Spielberger et al., 1983) and the Stanford Sleepiness Scale (Hoddes, Zarcone, Smythe, Phillips, \& Dement, 1973) directly before the MRI session to control for state levels of anxiety and sleepiness.

\section{Polysomnography}

All participants were scheduled for two consecutive nights of PSG sleep monitoring with a standard laboratory procedure and PSG montage. Sleep was recorded on 24-channel Sagura EEG-polysomnographs for eight hours from 'lights out' (10 to $11 \mathrm{pm})$ until 'lights on' (6 to 7 am). All recordings included EEG (C3-A2; C4-A1), EOG (horizontal and vertical) and EMG (submental), and were scored visually by experienced raters according to the criteria of the American Academy of Sleep Medicine (2007). During the adaptation night, all participants were screened for apneas and periodic leg movements by monitoring abdominal and thoracic effort, nasal airflow, oxymetry, and bilateral tibialis anterior EMG. Sleep recordings were evaluated for the following parameters of sleep continuity: total sleep time (TST); sleep efficiency (ratio of TST to time in bed in \%); sleep onset latency (SOL) defined as time from lights out until sleep onset (defined as first epoch of stage 2); wake after sleep onset (WASO) defined as difference between sleep period time (SPT; time from sleep onset until final awakening) and TST; number of awakenings; and arousal index per TST. Sleep architecture

parameters were amounts of stages 1, 2, slow wave sleep (SWS), and rapid eye movement sleep (REM) as percentage of SPT. 


\section{Sleep-fMRI experiment}

After the PSG assessment, the participants scheduled an appointment for an MRI session, which included, in this order, the emo-fMRI experiment described in Baglioni et al. (2014a), the sleep-fMRI experiment described here, the acquisition of T1-weighted MRI data (Spiegelhalder et al., 2013), and the acquisition of DTI data (Spiegelhalder et al., 2014).. The MRI sessions were conducted in the evening (starting between 18:00 and 20:00) at the Department of Radiology of the University of Freiburg Medical Center.

In the sleep-fMRI experiment, which had a total duration of $12 \mathrm{~min}$, sleep-related and control stimuli were presented in a block design ( 8 blocks with sleep-related stimuli, 16 blocks with control stimuli). Each block contained of either 5 sleep-related or 5 control stimuli that were presented for $3 \mathrm{~s}$ each, resulting in a block duration of $15 \mathrm{~s}$. Stimuli were composed of 10 sleep-related words and 20 control words (see Table 1) and were a subset of those used in previous own studies on sleep-related attentional bias (Spiegelhalder et al., 2008; 2009; 2010). Both word sets were matched carefully in terms of word length, syllables, word type and frequency of occurrence in the German language. Words were presented in each of four colors (red, green, blue, yellow) resulting in 120 experimental trials. These were randomly presented with the constraints that the same word or color would not appear twice in a row, the same word would not appear twice in the same block and sleep-related and control words would not appear together in the same block. Participants were instructed to passively view the stimuli. Successive blocks were separated by a simple reaction time task to ensure participants' attention during the whole scanning period. Specifically, a 3-cm grey solid circle was presented on black background for $15 \mathrm{~s}$ and was replaced at irregular intervals by a red 3-cm solid circle (on average 3 times per $15 \mathrm{~s}$ ). Participants were asked to respond as quickly as possible to the appearance of the red solid circle by pressing a button with the right index finger. All words as well as the simple reaction time task were presented on a screen behind 
the participants in the scanner bore using the Presentation ${ }^{\circledR}$ software (Neurobehavioral systems, http://www.neurobs.com/) and were visible via a tilted mirror positioned in front of the participants' eyes.

\section{MRI aquisition and analysis}

Functional echo-planar images (295 scans; data matrix: $64 \times 64$; voxel size $3 \mathrm{~mm} \times 3 \mathrm{~mm} \times 3$ mm; TE: $30 \mathrm{~ms}$; TR: $2.49 \mathrm{~s}$; motion and distortion correction by scanner software) and anatomical images (MPRAGE; data matrix: $256 \times 256$; voxel size $1 \mathrm{~mm} \times 1 \mathrm{~mm} \times 1 \mathrm{~mm}$; TE: 2.6 ms; TR: 2.2 s) were acquired using a 3 Tesla scanner (TIM-Trio, Siemens, Erlangen, Germany) with an 8-element head coil in a circularly polarized mode. The data was analyzed using the AFNI software (Cox, 1996). Preprocessing included slice time correction, coregistration with the anatomical image, normalisation (including resampling to $1 \mathrm{~mm} \times 1$ $\mathrm{mm} \times 1 \mathrm{~mm}$ voxels), smoothing (FWHM, $9 \mathrm{~mm}$ ), and filtering with a 1/128 Hz high-pass filter.

First-level analyses were performed for each subject with the following regressors that were convolved with a standard hemodynamic response function: sleep-related words, control words (each as a block design regressor with a duration of $15 \mathrm{~s}$ per block) and responses to the reaction time task. Additionally, the six head movement parameters that were estimated during the online motion correction were used as covariates of no interest. Afterwards, individual difference maps were calculated by subtracting the regression coefficients of the 'control words' regressor from the regression coefficients of the 'sleep-related words' regressor. In the second-level analysis, between-group differences in the contrast 'sleeprelated words vs. control words' were investigated using whole-brain voxel-wise independent t-tests. For ensuring data and analysis quality, brain activity related to reading (presentation of any words) and responses to the reaction time task were also analysed in additional whole- 
brain second-level analyses. A Monte-Carlo simulation was performed to estimate the probability of false positive clusters for the whole-brain analyses. According to this, overall statistical significance of $p<0.05$ was obtained by considering cluster sizes above $1696 \mathrm{~mm}^{3}$ at a voxel threshold of $p<0.005(\mathrm{~T}>$ 2.92). MRIcron (http://www.mricro.com/mricron/install.html) was used for three-dimensional visualisation of the results on a ch2better.nii.gz atlas.

Exploratory region-of-interest analyses were carried out for anxiety-related brain areas, namely the left and right amygdala and insula (Etkin \& Wagner, 2007), as well as for craving-related brain areas, namely the left and right ventral striatum and PCC (Schacht et al., 2012). For these analyses, individual region-of-interest masks were created by using the FreeSurfer software (http://surfer.nmr.mgh.harvard.edu/). For each participant, the mean values for the 'sleep-related words vs. control words' contrast of all voxels in the above mentioned brain areas were calculated and subsequently analysed using multiple independent t-tests. The unadjusted level of significance was set at $p<0.05$ (two-tailed) for these analyses.

\section{Stroop task}

After the MRI session, an emotional Stroop task was administered outside the scanner using the Presentation ${ }^{\circledR}$ software (Neurobehavioral systems, http://www.neurobs.com/). In each trial, either a sleep-related or a control word was presented in the center of a computer monitor, in one of four possible colors (red, green, blue, yellow). Participants were instructed to respond to the color of the words by pressing a button of corresponding color with the index finger of their dominant writing hand on a designated button box. The stimulus set was identical to the one used in the sleep-fMRI experiment before (see Table 1). After 24 practice trials (using additional neutral words), the 120 experimental trials were randomly presented 
in blocks of 5 sleep-related or control words with the same constraints that were used for the sleep-fMRI experiment.

All trials with errors were excluded from the analyses. Additionally, response times that deviated from the persons' mean by more than \pm 3 standard deviations were eliminated as outliers. Attentional bias scores were calculated for each participant as the mean response latency to sleep-related words minus the mean response latency to control words. Positive attentional bias scores indicate vigilance for sleep-related words because performance is expected to be disrupted by processing of concern-related information. The between-group difference in attentional bias scores was analysed using an independent t-test with statistical significance at $p<0.05$ (2-tailed).

(Please insert Table 1 here)

\section{Results}

\section{Sample characteristics}

Demographic, sleep and daytime functioning variables for patients with chronic insomnia and good sleeper controls are shown in Table 2. There were no significant group differences in sex distribution, age or body mass index (BMI). Insomnia patients had significantly higher scores on the ISI, PSQI, DBAS-16, GSES, and BDI, as well as on the cognitive subscale of the PSAS and the trait anxiety subscale of the STAI. Of note, BDI scores were not significantly different between groups after excluding the two sleep-related items [insomnia patients: $4.6 \pm 3.6$, good sleeper controls: $3.1 \pm 2.9, t(53)=1.64, p=0.111]$. There were no significant group differences for the somatic subscale of the PSAS, the ESS, the SSS and the 
state anxiety subscale of the STAI. Two patients suffered from sleep-onset insomnia, 6 from sleep-maintenance insomnia, 1 from non-restorative sleep, and 11 from mixed insomnia. The average duration of insomnia was $9.5 \pm 9.2$ years (range 1-30 years). Nine patients have never used sleep medication, 5 patients have used benzodiazepines or benzodiazepine receptor agonists, 7 patients have used sedating antidepressants, one patient has used a sedating antipsychotic, and 3 patients have used melatonin (four patients have used medication from more than one drug class).

(Please insert Table 2 here)

\section{Polysomnography}

The polysomnographic data are presented in Table 3. In both nights, patients with insomnia had a significantly lower total sleep time and sleep efficiency, lower REM \% as well as increased WASO compared to good sleeper controls. In addition, patients with insomnia had a significantly reduced stage $2 \%$ in the second night.

(Please insert Table 3 here)

\section{fMRI results}

The whole-brain analysis of the 'sleep-related words vs. control words' contrast did not show any significant cluster of voxels with between-group differences. The same result was obtained when BDI and STAI trait scores were used as covariates in the whole-brain analysis. The results of the whole-brain analysis of brain activity related to reading and responses to the reaction time task are presented in the Supplementary material (figures S1 and S2). Reading was associated with increased brain activity in a network encompassing the primary 
and extrastriate visual cortex as well as the left angular gyrus. Responses to the reaction time task were associated with bilateral activation of the sensorimotor cortex including primary, premotor and supplementary motor areas, as well as with activation of cerebellar areas and the orbitofrontal cortex.

The results of the exploratory region-of-interest analyses of the 'sleep-related words vs. control words' contrast are presented in Figure 1. These analyses did not show any significant between-group differences [left amygdala: $t(53)=-0.11, p=0.911$; right amygdala: $t(53)=$ $-0.60, p=0.551$; left insula: $t(53)=0.35, p=0.724$; right insula: $t(53)=0.83, p=0.414$; left ventral striatum: $t(53)=0.81, p=0.423$; right ventral striatum: $t(53)=1.15, p=0.257$; left PCC: $t(53)=-0.12, p=0.908$; right PCC: $t(53)=-0.00, p=1.000]$. The between-group differences remained non-significant when BDI and STAI trait scores were used as covariates in the analyses. In addition, exploratory analyses did not show any significant correlations between these region-of-interest-based values and questionnaire scores/ PSG parameters of the second sleep laboratory night.

(Please insert Figure 1 here)

\section{Stroop task}

Mean reaction times were not significantly different between groups [insomnia patients: 802 $\pm 112 \mathrm{~ms}$, good sleeper controls: $772 \pm 80 \mathrm{~ms}, t(53)=1.05, p=0.303]$. Data loss included $1.0 \%$ of data due to errors, $0.7 \%$ in the insomnia group and $1.2 \%$ in good sleeper controls $[t(53)=-1.06, p=0.296]$. An additional $1.0 \%$ of data was excluded due to outlier identification (1.0\% in both groups). Attentional bias scores were not significantly different between groups [insomnia patients: $4.6 \pm 20.4 \mathrm{~ms}$, good sleeper controls: $4.5 \pm 28.7 \mathrm{~ms}, t(53)$ $=0.01, p=0.994]$. This result remained non-significant after the inclusion of BDI and STAI 
scores as covariates in the analysis. In addition, exploratory analyses did not show any significant correlations between attentional bias scores and questionnaire scores/ PSG parameters of the second sleep laboratory night.

\section{Discussion}

Contrary to the hypotheses of the current study, patients with chronic insomnia did not differ from good sleeper controls in terms of brain reactivity and selective attention to sleep-related words. Data and analysis quality of this study were ensured by additional analyses which revealed the expected pattern of brain activity associated with reading and finger movements in a reaction time task (for comparison, see Kim et al., 1993; Price, 2012; Rowe \& Siebner, 2012).

\section{Strengths of the study}

What are potential reasons for this pattern of negative findings? The current study included a well-characterised drug-free sample of clinically referred insomnia patients who were rigorously screened to eliminate any comorbid medical or psychiatric disorder. Both psychometric and polysomnographic data of the participants were comparable to previous studies of patients with insomnia and good sleeper controls (Baglioni et al., 2014b). Like in many previous studies (e.g. Fortier-Brochu \& Morin, 2014), depression and trait anxiety scores were increased in the insomnia group. However, none of our participants had a clinically significant affective or anxiety disorder. Thus, it appears to be unlikely that the sample composition is a major factor explaining the pattern of negative findings. 
The stimuli of the current study were a subset of those used in two previous investigations, that showed a sleep-related attentional bias in patients with insomnia compared to good sleeper controls (Spiegelhalder et al., 2010), and a significant positive association between PSQI scores and sleep-related attentional bias in non-clinical individuals (Spiegelhalder et al., 2009). The 10 sleep-related words were the same as in these former studies, and the 20 control words of the current study were a subset of the 30 control words of the two previous investigations. Moreover, in comparison to the randomised stimulus presentation in earlier studies (Barclay \& Ellis, 2013; Spiegelhalder et al., 2008; 2009; 2010; Taylor et al., 2003), the blockwise presentation in the current study should be expected to lead to increased effect sizes (Salo, Henik, \& Robertson, 2001; Waters \& Feyerabend, 2000).

\section{Power considerations}

Although the sample size of this study is currently one of the largest ones of an fMRI study in the insomnia field, a power analysis suggests that only effect sizes of at least Cohen's $d=$ 0.80 could have been detected at an alpha level of $p<0.05$ with a power of $80 \%$. However, the largest reported effect size of studies that used words for investigating sleep-related attentional bias was Cohen's $d=0.67$ (MacMahon et al., 2006), which would result in an achieved power of only $68 \%$ for the current study (for an overview of attentional bias studies in insomnia including sample sizes and effect sizes please refer to Table 2 in Harris et al., 2015). Moreover, the largest effect size that was reported in a study using the emotional Stroop task in insomnia patients was Cohen's $d=0.52$ (Spiegelhalder et al., 2010), which would result in an achieved power of $45 \%$. At the outset of the experiment, it was the hope that fMRI may be more sensitive to alterations in sleep-related information processing than behavioural measures, however, the power analysis still illustrates that false-negative findings are currently inevitable in this field. Moreover, as the current sample of insomnia patients was not significantly different from healthy controls in the emotional Stroop task, it may have 
been particularly unlikely to obtain significant group differences in the neurobiological domain. Still, it is highly important to report negative findings in order to inform future studies about the true size of potential effects. This said, the negative finding of the current study lowers the effect size that would be achieved by meta-analysis of sleep-related attentional bias effects in insomnia patients compared to good sleeper controls. Thus, the results from the present study call into question that attentional bias to sleep-related stimuli is a core feature of chronic insomnia.

\section{Limitations}

It has been questioned whether words have a sufficiently high potential to attract attention compared to pictorial stimuli (Moritz et al., 2008). In line with this, there are now four studies (including the current one) that failed to find significant differences between patients with insomnia/ poor sleepers and good sleeper controls using the emotional Stroop task (Barclay \& Ellis, 2013; Lundh et al., 1997; Spiegelhalder et al., 2008). Moreover, the current study may have been particularly likely to yield small effect sizes because most of the participants completed the emo-fMRI experiment, in which negatively valenced pictorial insomniaspecific stimuli were presented to them directly before the sleep-fMRI experiment (Baglioni et al., 2014a). However, if patients with chronic insomnia adapt so quickly to the presentation of sleep-related stimuli, this may question the clinical significance of computerised tasks measuring sleep-related attentional bias.

Some further limitations of the current study have to be acknowledged. First, fatigue may have affected our results given that the experiments were conducted in the evening and the emo-fMRI experiment described in Baglioni et al. (2014) was performed before the current one. Thus, it would have been helpful to assess fatigue or sleepiness both before and after the MRI session. Second, the healthy controls of the current study showed comparably poor sleep 
in contrast to some previous investigations, especially with respect to WASO and the number of awakenings. However, a mean sleep efficiency below $90 \%$ is not an uncommon finding in polysomnographic investigations of middle-aged healthy samples (Ohayon, Carskadon, Guilleminault, \& Vitiello, 2004). Third, several variables that may have affected the current results were not assessed, including chronotype, duration of the current episode of insomnia, race or ethnicity, and ....

\section{Outlook}

In summary, the current study failed to find between-group differences in brain reactivity and selective attention to sleep-related words. Future studies may use pictorial stimuli and larger sample sizes for investigating sleep-related information processing in chronic insomnia.

\section{Acknowledgements}

Dr. Baglioni and Dr. Riemann have received funding from the European Community's Seventh Framework Programme (People, Marie Curie Actions, Intra-European Fellowship, FP7-PEOPLE-IEF-2008) under grant agreement number 235321 for this work. 


\section{References}

American Academy of Sleep Medicine. The AASM Manual for the Scoring of Sleep and Associated Events: Rules, Terminology and Technical Specifications. Westchester: Author.

American Psychiatric Association (2000). Diagnostic and Statistical Manual of Mental Disorders (4th ed., text rev.). Washington, DC: Author.

Baglioni, C., Lombardo, C., Bux, E., Hansen, S., Salveta, C., Biello, S., ... Espie, C. A. (2010). Psychophysiological reactivity to sleep-related emotional stimuli in primary insomnia. Behaviour Research and Therapy, 48, 467-475.

Baglioni, C., Spiegelhalder, K., Regen, W., Feige, B., Nissen, C., Lombardo, C., ... Riemann, D. (2014a). Insomnia disorder is associated with increased amygdala reactivity to insomnia-related stimuli. Sleep, 37, 1907-1917.

Baglioni, C., Regen, W., Teghen, A., Spiegelhalder, K., Feige, B., Nissen, C., \& Riemann, D. (2014b). Sleep changes in the disorder of insomnia: a meta-analysis of polysomnographic studies. Sleep Medicine Reviews, 18, 195-213.

Barclay, N. L., \& Ellis, J. G. (2013). Sleep-related attentional bias in poor versus good sleepers is independent of affective valence. Journal of Sleep Research, 22, 414-421.

Bastien, C. H., Vallières, A., \& Morin, C. M. (2001). Validation of the Insomnia Severity Index as an outcome measure for insomnia research. Sleep Medicine, 2, 297-307.

Beck, A. T., \& Steer, R. A. (1987). Beck Depression Inventory. San Antonio: The Psychological Corporation.

Broomfield, N. M., \& Espie, C. A. (2005). Towards a valid, reliable measure of sleep effort. Journal of Sleep Research, 14, 401-407. 
Buysse, D. J., Reynolds, C. F. $3^{\text {rd }}$, Monk, T. H., Berman, S. R., \& Kupfer, D. J. (1989). The Pittsburgh Sleep Quality Index: a new instrument for psychiatric practice and research. Psychiatry Research, 28, 193-213.

Cox, R. W. (1996). AFNI: software for analysis and visualization of functional magnetic resonance neuroimages. Computers and Biomedical Research, 29, 162-173.

Edinger, J. D., Bonnet, M. H., Bootzin, R. R., Doghramji, K., Dorsey, C. M., Espie, C. A., ... Stepanski, E. J. (2004). Derivation of research diagnostic criteria for insomnia: report of an American Academy of Sleep Medicine Work Group. Sleep, 27, 1567-1596.

Espie, C. A., Broomfield, N. M., MacMahon, K. M., Macphee, L. M., \& Taylor, L. M. (2006). The attention-intention-effort pathway in the development of psychophysiologic insomnia: a theoretical review. Sleep Medicine Reviews, 10, 215-245.

Etkin, A., \& Wagner, T. D. (2007). Functional neuroimaging of anxiety: a meta-analysis of emotional processing in PTSD, social anxiety disorder, and specific phobia. American Journal of Psychiatry, 164, 1476-1488.

Fortier-Brochu, E., \& Morin, C. M. (2014). Cognitive impairment in individuals with insomnia: clinical significance and correlates. Sleep, 37, 1787-1798.

Harvey, A. G. (2002). A cognitive model of insomnia. Behaviour Research and Therapy, 40, 869-893.

Hoddes, E., Zarcone, V., Smythe, H., Phillips, R., \& Dement, W. C. (1973). Quantification of sleepiness: a new approach. Psychophysiology, 10, 431-436.

Jansson-Fröjmark, M., Bermas, M., \& Kjellen, A. (2013). Attentional bias in insomnia: the dot-probe task with pictorial stimuli depicting daytime fatigue/ malaise. Cognitive Therapy and Research, 37, 534-546.

Johns, M. W. (1991). A new method for measuring daytime sleepiness: the Epworth sleepiness scale. Sleep, 14, 540-545. 
Jones, B. T., Macphee, L. M., Broomfield, N. M., Jones, B. C., \& Espie, C. A. (2005). Sleeprelated attentional bias in good, moderate, and poor (primary insomnia) sleepers. Journal of Abnormal Psychology, 114, 249-258.

Kim, S. G., Ashe, J., Hendrich, K., Ellermann, J. M., Merkle, H., Ugurbil, K., Georgopoulos, A. P. (1993). Functional magnetic resonance imaging of motor cortex: hemispheric asymmetry and handedness. Science, 261, 615-617.

Lombardo, C., Battagliese, G., David, M., Lorusso, B., Baglioni, C., Espie, C., \& Violani, C. (2013). Psychophysiological reactivity to symptom-related emotional stimuli in insomnia: a replication and extension to disordered eating. Sleep and Biological Rhythms, 11, 20-28.

Lundh, L. G., Fröding, A., Gyllenhammar, L., Broman, J. E., \& Hetta, J. (1997). Cognitive bias and memory performance in patients with persistent insomnia. Scandinavian Journal of Behaviour Therapy, 26, 27-35.

MacMahon, K. M., Broomfield, N. M., \& Espie, C. A. (2006). Attention bias for sleeprelated stimuli in primary insomnia and delayed sleep phase syndrome using the dot-probe task. Sleep, 29, 1420-1427.

Morin, C. M., Vallières, A., \& Ivers, H. (2007). Dysfunctional beliefs and attitudes about sleep (DBAS): validation of a brief version (DBAS-16). Sleep, 30, 1547-1554.

Moritz, S., Fischer, B. K., Hottenrott, B., Kellner, M., Fricke, S., Randjbar, S., Jelinek, L. (2008). Words may not be enough! No increased emotional Stroop effect in obsessivecompulsive disorder. Behaviour Research and Therapy, 46, 1101-1104.

Nicassio, P. M., Mendlowitz, D. R., Fussell, J. J., \& Petras, L. (1985). The phenomenology of the pre-sleep state: the development of the pre-sleep arousal scale. Behaviour Research and Therapy, 23, 263-271.

Ohayon, M. M., Carskadon, M. A., Guilleminault, C., \& Vitiello, M. V. (2004). Metaanalysis of quantitative sleep parameters from childhood to old age in healthy individuals: developing normative sleep values across the human lifespan. Sleep, 27, 1255-1273. Price, 
C. J. (2012). A review and synthesis of the first 20 years of PET and fMRI studies of heard speech, spoken language and reading. Neuroimage, 62, 816-847.

Rowe, J. B., \& Siebner, H. R. (2012). The motor system and its disorders. Neuroimage, 61, 464-477.

Sagaspe, P., Sanchez-Ortuno, M., Charles, A., Taillard, J., Valtat, C., Bioulac, B., \& Philip, P. (2006). Effects of sleep deprivation on color-word, emotional, and specific stroop interference and on self-reported anxiety. Brain and Cognition, 60, 76-87.

Salo, R., Henik, A., \& Robertson, L.C. (2001). Interpreting Stroop interference: an analysis of differences between task versions. Neuropsychology, 15, 462-471.

Schacht, J. P., Anton, R. F., \& Myrick, H. (2012). Functional neuroimaging studies of alcohol cue reactivity: a quantitative meta-analysis and systematic review. Addiction Biology, 18, 121-133.

Spiegelhalder, K., Espie, C., Nissen, C., \& Riemann, D. (2008). Sleep-related attentional bias in patients with primary insomnia compared with sleep experts and healthy controls. Journal of Sleep Research, 17, 191-196.

Spiegelhalder, K., Espie, C., \& Riemann, D. (2009). Is sleep-related attentional bias due to sleepiness or sleeplessness? Cognition and Emotion, 23, 541-550.

Spiegelhalder, K., Kyle, S. D., Feige, B., Prem, M., Nissen, C., Espie, C. A., \& Riemann, D. (2010). The impact of sleep-related attentional bias on polyomnographically measured sleep in primary insomnia. Sleep, 33, 107-112.

Spiegelhalder, K., Regen, W., Baglioni, C., Klöppel, S., Abdulkadir, A., Hennig, J., ... Feige, B. (2013). Insomnia does not appear to be associated with substantial structural brain changes. Sleep, 36, 731-737.

Spiegelhalder, K., Regen, W., Prem, M., Baglioni, C., Nissen, C., Feige, B., ... Riemann, D. (2014). Reduced anterior internal capsule white matter integrity in primary insomnia. Human Brain Mapping, 35, 3431-3438. 
Spielberger, C. D., Gorsuch, R. L., Lushene, R., Vogg, P. R., \& Jacobs, G. A. (1983). Manual for the state-trait anxiety inventory. Palo Alto, Consulting Psychologists Press.

Taylor, L. M., Espie, C. A., \& White, C. A. (2003). Attentional bias in people with acute versus persistent insomnia secondary to cancer. Behavioral Sleep Medicine, 1, 200-212.

Williams, J. M., Mathews, A., \& MacLeod, C. (1996). The emotional Stroop task and psychopathology. Psychological Bulletin, 120, 3-24.

Woods, H., Marchetti, L. M., Biello, S. M., \& Espie, C. A. (2009). The clock as a focus of selective attention in those with primary insomnia: an experimental study using a modified Posner paradigm. Behaviour Research and Therapy, 47, 231-236.

Waters, A. J., \& Feyerabend, C. (2000). Determinants and effects of attentional bias in smokers. Psychology of Addictive Behaviors, 14, 111-120.

Harris, K., Spiegelhalder, K., Espie, C. A., MacMahon, K. M., Woods, H. C., \& Kyle, S. D. (2015). Sleep-related attentional bias in insomnia: A state-of-the-science review. Clinical Psychology Review, 42, 16-27. 


\section{Figure legends}

Figure 1: Mean $\beta$ values for the contrast 'sleep-related words vs. control words' in the amygdala, insula, ventral striatum and posterior cingulate cortex (PCC). Blue triangles represent patients with chronic insomnia, black dots represent good sleeper controls. No significant group differences were found in any of these exploratory region-of-interest analyses. Horizontal bars represent group means.

Figure S1: Brain areas with significantly increased activation during reading (minimal cluster size of $1696 \mathrm{~mm}^{3}$; voxel threshold of $\left.p<0.005\right)$.

Figure S2: Brain areas with significantly increased activation during responses to the reaction time task/ finger movements (minimal cluster size of $1696 \mathrm{~mm}^{3}$; voxel threshold of $p$ $<0.005)$. 


\section{Tables}

Table 1: Words used in the sleep-fMRI experiment and in the emotional Stroop task.

\begin{tabular}{lll} 
Sleep-related words & Control words & \\
\hline müde (tired) & nahe (close) & ewig (forever) \\
Bett (bed) & Brot (bread) & Baum (tree) \\
wach (awake) & brav (well-behaved) & toll (amazing) \\
Kopfkissen (pillow) & Holzbalken (wood truss) & Instrument (instrument) \\
Bettdecke (sheets) & Grundlage (basis) & Umleitung (route diversion) \\
schnarchen (to snore) & schleudern (to toss) & schneidern (to tailor) \\
Nickerchen (nap) & Tapferkeit (courage) & Dachziegel (tile) \\
wecken (to rouse) & kleben (to glue) & wiegen (to weigh) \\
Nacht (night) & Stadt (city) & Stück (part) \\
Schlaf (sleep) & Frosch (frog) & Schirm (shield)
\end{tabular}


Table 2: Demographic and clinical characteristics of the study population (means \pm standard deviations). BMI: body mass index; ISI: Insomnia Severity Index; PSQI: Pittsburgh Sleep Quality Index; DBAS-16: brief version of the Dysfunctional Beliefs and Attitudes about Sleep Scale; GSES: Glasgow Sleep Effort Scale; PSAS: Pre-Sleep Arousal Scale; ESS: Epworth Sleepiness Scale; SSS: Stanford Sleepiness Scale; BDI: Beck Depression Inventory; STAI, State-Trait Anxiety Inventory. *: $p<0.05 ; * *: p<0.01 ; * * *: p<0.001$.

\begin{tabular}{|c|c|c|c|c|c|c|c|c|c|}
\hline & \multicolumn{3}{|c|}{ Patients } & \multicolumn{3}{|c|}{ Controls } & $t / x^{2}$ & \multicolumn{2}{|l|}{$p$} \\
\hline $\operatorname{Sex}[F / M]$ & \multicolumn{3}{|c|}{$10 / 10$} & \multicolumn{3}{|c|}{$21 / 14$} & 0.52 & 0.472 & \\
\hline Age [years] & 42.6 & \pm & 14.9 & 40.0 & \pm & 9.1 & 0.79 & 0.430 & \\
\hline BMI $\left[\mathrm{kg} / \mathrm{m}^{2}\right]$ & 23.4 & \pm & 2.0 & 22.9 & \pm & 3.2 & 0.66 & 0.515 & \\
\hline$|S|$ & 14.7 & \pm & 3.9 & 2.2 & \pm & 2.3 & 14.97 & $<0.001$ & $* * *$ \\
\hline PSQI & 10.4 & \pm & 3.2 & 3.7 & \pm & 1.8 & 10.17 & $<0.001$ & $\star \star \star *$ \\
\hline DBAS-16 & 4.7 & \pm & 1.5 & 2.3 & \pm & 1.0 & 7.23 & $<0.001$ & $* * *$ \\
\hline GSES & 7.0 & \pm & 2.8 & 1.1 & \pm & 1.3 & 10.75 & $<0.001$ & $* * *$ \\
\hline PSAS - cognitive & 19.2 & \pm & 5.9 & 13.4 & \pm & 4.2 & 4.27 & $<0.001$ & $* * *$ \\
\hline PSAS - somatic & 12.1 & \pm & 4.2 & 10.3 & \pm & 3.0 & 1.75 & 0.085 & \\
\hline ESS & 7.6 & \pm & 4.8 & 6.8 & \pm & 3.9 & 0.65 & 0.518 & \\
\hline SSS & 2.3 & \pm & 1.1 & 2.2 & \pm & 0.9 & 0.03 & 0.980 & \\
\hline $\mathrm{BDI}$ & 7.2 & \pm & 4.1 & 3.6 & \pm & 3.3 & 3.57 & 0.001 & $* *$ \\
\hline STAI - state & 33.5 & \pm & 7.1 & 34.5 & \pm & 6.3 & -0.55 & 0.586 & \\
\hline STAI - trait & 39.4 & \pm & 8.4 & 33.1 & \pm & 7.3 & 2.88 & 0.006 & $\star *$ \\
\hline
\end{tabular}


Table 3: Polysomnographic data (means \pm standard deviations). TST: total sleep time; PLMS: periodic leg movements in sleep; SPT: sleep period time; SWS: slow wave sleep; REM: rapid eye movement sleep. *: $p<0.05 ; * *: p<0.01 ; * * *: p<0.001$.

\begin{tabular}{|c|c|c|c|c|c|}
\hline First Night: & Patients & Controls & $t$ & $p$ & \\
\hline Total sleep time [min] & $344.6 \pm 60.4$ & $387.7 \pm 44.4$ & -2.78 & 0.009 & ** \\
\hline Sleep efficiency [\%] & $72.0 \pm 12.6$ & $80.8 \pm 9.2$ & -2.72 & 0.011 & * \\
\hline Sleep onset latency [min] & $28.4 \pm 12.2$ & $20.2 \pm 19.0$ & 1.92 & 0.061 & \\
\hline Wake after sleep onset [min] & $92.5 \pm 53.5$ & $64.9 \pm 35.2$ & 2.06 & 0.049 & * \\
\hline Number of awakenings & $31.1 \pm 13.8$ & $38.8 \pm 14.8$ & -1.90 & 0.064 & \\
\hline Arousal index / TST [ $\left.\mathrm{h}^{-1}\right]$ & $19.3 \pm 7.5$ & $20.3 \pm 7.6$ & -0.47 & 0.638 & \\
\hline Sleep apnoea index / TST $\left[\mathrm{h}^{-1}\right]$ & $0.4 \pm 0.9$ & $0.4 \pm 0.7$ & -0.22 & 0.827 & \\
\hline PLMS arousal index / TST $\left[\mathrm{h}^{-1}\right]$ & $0.3 \pm 0.6$ & $0.4 \pm 0.8$ & -0.45 & 0.655 & \\
\hline Stage $1[\%$ SPT] & $9.9 \pm 4.7$ & $11.2 \pm 5.0$ & -0.99 & 0.328 & \\
\hline Stage 2 [\% SPT] & $46.9 \pm 9.9$ & $49.8 \pm 8.4$ & -1.13 & 0.268 & \\
\hline SWS [\% SPT] & $8.2 \pm 7.7$ & $7.1 \pm 6.8$ & 0.53 & 0.800 & \\
\hline REM [\% SPT] & $14.1 \pm 3.1$ & $17.5 \pm 4.9$ & -3.12 & 0.003 & ** \\
\hline Second Night: & Patients & Controls & $t$ & $p$ & \\
\hline Total sleep time [min] & $373.4 \pm 66.9$ & $418.0 \pm 21.9$ & -3.64 & 0.001 & ** \\
\hline Sleep efficiency [\%] & $77.9 \pm 14.1$ & $87.1 \pm 4.5$ & -3.58 & 0.001 & ** \\
\hline Sleep onset latency [min] & $17.8 \pm 10.9$ & $15.4 \pm 12.2$ & 0.74 & 0.463 & \\
\hline Wake after sleep onset [min] & $77.5 \pm 62.2$ & $41.9 \pm 17.3$ & 3.20 & 0.002 & $\star *$ \\
\hline Number of awakenings & $33.7 \pm 17.9$ & $35.2 \pm 13.9$ & -0.35 & 0.730 & \\
\hline Arousal index / TST $\left[\mathrm{h}^{-1}\right]$ & $17.2 \pm 6.4$ & $15.8 \pm 6.5$ & 0.77 & 0.447 & \\
\hline Stage $1[\%$ SPT] & $8.2 \pm 4.0$ & $8.8 \pm 4.6$ & -0.44 & 0.660 & \\
\hline Stage 2 [\% SPT] & $47.9 \pm 10.8$ & $53.6 \pm 6.0$ & -2.54 & 0.014 & * \\
\hline SWS [\% SPT] & $10.5 \pm 8.9$ & $9.2 \pm 7.5$ & 0.60 & 0.552 & \\
\hline REM [\% SPT] & $16.1 \pm 6.5$ & $19.4 \pm 3.9$ & -2.34 & 0.023 & * \\
\hline
\end{tabular}

